\title{
Compromise of Auditory Cortical Tuning and Topography after Cross-Modal Invasion by Visual Inputs
}

\author{
Yu-Ting Mao and Sarah L. Pallas \\ Department of Biology and Neuroscience Institute, Georgia State University, Atlanta, Georgia 30303
}

Brain damage resulting in loss of sensory stimulation can induce reorganization of sensory maps in cerebral cortex. Previous research on recovery from brain damage has focused primarily on adaptive plasticity within the affected modality. Less attention has been paid to maladaptive plasticity that may arise as a result of ectopic innervation from other modalities. Using ferrets in which neonatal midbrain damage results in diversion of retinal projections to the auditory thalamus, we investigated how auditory cortical function is impacted by the resulting ectopic visual activation. We found that, although auditory neurons in cross-modal auditory cortex (XMAC) retained sound frequency tuning, their thresholds were increased, their tuning was broader, and tonotopic order in their frequency maps was disturbed. Multisensory neurons in XMAC also exhibited frequency tuning, but they had longer latencies than normal auditory neurons, suggesting they arise from multisynaptic, non-geniculocortical sources. In a control group of animals with neonatal deafferentation of auditory thalamus but without redirection of retinal axons, tonotopic order and sharp tuning curves were seen, indicating that this aspect of auditory function had developed normally. This result shows that the compromised auditory function in XMAC results from invasion by ectopic visual inputs and not from deafferentation. These findings suggest that the cross-modal plasticity that commonly occurs after loss of sensory input can significantly interfere with recovery from brain damage and that mitigation of maladaptive effects is critical to maximizing the potential for recovery.

\section{Introduction}

Alterations of sensory inputs can result in plastic changes to sensory pathways. Research on within-modality plasticity after sensory manipulations has provided important information about how sensory systems compensate for a loss of input (for review, see Buonomano and Merzenich, 1998). However, recovery can involve multiple brain areas and sensory modalities (for review, see Kral and Sharma, 2012). For example, visual areas can become responsive to sound stimulation in blind animals (Rauschecker, 1995; Izraeli et al., 2002; Piché et al., 2004), and recordings from deaf animals show visual responses in auditory regions (Kral, 2007; Lomber et al., 2010). Although cross-modal (XM) plasticity is a common outcome of sensory loss, it has received considerably less study than unimodal plasticity.

The success of clinical interventions after damage to sensory pathways can be negatively affected by XM plasticity. Research on deaf patients (Sharma et al., 2007, 2009) shows that auditory

\footnotetext{
Received Dec. 12, 2011; revised May 23, 2012; accepted May 28, 2012.

Author contributions: Y.-T.M. and S.L.P. designed research; Y.-T.M. performed research; Y.-T.M. analyzed data; Y.-T.M. and S.L.P. wrote the paper.

This research was supported by National Science Foundation Grant IBN-0451018, National Institutes of Health Grant EY/MH 12696, and the Georgia State University Research Foundation (all to S.L.P), a Georgia State University Brains and Behavior scholarship (Y.T.M.), and National Science Foundation Grant IBN-9876754 under the Science and Technology Centers Program. We thank Profs. Khaleel A. Razak, Robert Liu, Donald Edwards, and Charles Derby for their comments in the preparation of this manuscript. We also thank Prof. Jennifer Bizley and Dr. Jeff Ko for advice on ferret anesthesia and Prof. Jennifer Bizley and Tim Balmer for help with MATLAB code. We are indebted to the Department of Animal Resources at Georgia State University and particularly Cindy Marshall for expert animal care.

Correspondence should be addressed to Sarah L. Pallas, Department of Biology and Neuroscience Institute, Georgia State University, P.0. Box 5030, Atlanta, GA 30303. E-mail: spallas@gsu.edu.

DOI:10.1523/JNEUROSCI.6524-11.2012

Copyright $\odot 2012$ the authors $\quad 0270-6474 / 12 / 3210338-14 \$ 15.00 / 0$
}

cortices have often received XM projections by the time that cochlear prostheses can be implanted, interfering with the success of the implants (Lee et al., 2001). These findings point out the importance of studying XM interactions for purposes of optimizing rehabilitation. Animal models using deafness have yielded crucial information (Kral, 2007; Lomber et al., 2010) but do not allow study of competitive interactions between auditory and $\mathrm{XM}$ visual inputs to AC. Our approach of partial midbrain damage reroutes retinal axons to auditory thalamus, placing visual and auditory inputs in a controlled state of competition (Sur et al., 1988; Pallas et al., 1999; Mao et al., 2011) while still allowing sensory activation of both modalities, thus enabling study of the effects of different degrees of XM plasticity on residual auditory function.

We reported previously that auditory cortex (AC) of ferrets after unilateral neonatal midbrain damage contains neurons that respond to a single modality (auditory and visual neurons) and to both modalities (bisensory neurons). Because these different response types are distributed randomly within cross-modal auditory cortex (XMAC) rather than in a segregated manner, communication between unimodal neurons might be disrupted by neighboring XM neurons. We tested whether competition from ectopic visual inputs compromises auditory tuning and topography in XMAC using in vivo singleunit recordings in anesthetized ferrets.

We found that auditory tuning and tonotopic mapping in auditory cortex are impaired after XM plasticity. Because the same neonatal midbrain lesions coupled with enucleation of both eyes did not produce the effects, we argue that the compromised function results specifically from the ectopic visual inputs. Inter- 
estingly, early enucleation by itself increased auditory sensitivity. These results provide support for the hypothesis that competition between auditory and visual inputs after XM plasticity underlies the compromised auditory function in patients with partial hearing loss, and they also provide a potential explanation for the improved auditory function often observed in blind humans (Bavelier and Neville, 2002).

Preliminary results from some of these experiments have been published previously in abstract form (Mao and Pallas, 2010).

\section{Materials and Methods}

Animals. In total, 26 adult pigmented ferrets (Mustela putorius furo) of either sex were included in this study. Timed pregnant ferrets were either obtained from Marshall Farms 2 weeks before parturition or bred in the Georgia State University facility and were kept on a 14/10 h light/dark cycle. Kits were weaned at $6-8$ weeks of age. Nonlactating adults were fed Marshall Farms ferret diet and kept on a $12 \mathrm{~h}$ light/dark cycle. Animals were divided into normal $(n=8)$, XM $(n=9)$, blind $(n=3)$, and blind-lesioned groups $(n=6)$. Both XM and blind-lesioned groups were subjected to neonatal surgeries. All animals were treated in accordance with protocols approved by the Institutional Animal Care and Use Committee at Georgia State University and met or exceeded standards of care established by the United States Department of Agriculture and the Society for Neuroscience.

Neonatal surgery. Surgical procedures to induce XM plasticity were similar to those described previously (Pallas et al., 1999; Mao et al., 2011). All invasive procedures were performed under sterile conditions. Within $12 \mathrm{~h}$ after birth, ferret kits were anesthetized by isoflurane $(1-4 \%$ as needed). The skull overlying the midbrain was exposed and removed through an incision in the skin. The superficial, retinorecipient layers of the superior colliculus (sSC) primarily on the left side and the central nucleus of the inferior colliculi (ICc) on both sides were then cauterized to varying extents, and the brachium of the left IC was severed. The incision was closed using surgical adhesive (VetBond; $3 \mathrm{M}$ ). For generating blind animals, both eyes were enucleated within the first postnatal week (the eyes open at approximately P30 in ferrets). The surgical site was cleaned and draped. The lids were opened along the future eyelid margin (visible at this age) by a scalpel or a microscissor. The orbits were separated from the surrounding conjunctiva and were completely removed, taking care to eliminate all pigmented epithelium that could conceivably regenerate photoreceptors. Typically, there was no bleeding during this procedure. The eyelids were reclosed with tissue glue. To generate blind-lesioned animals, the two procedures were combined. After surgery, the kits were given subcutaneous fluids and a respiratory stimulant ( $2 \mathrm{mg} / \mathrm{kg}$ doxapram, s.q.) and warmed on a heating pad. Kits were returned to their dam after they recovered from anesthesia. Analgesics $(0.01 \mathrm{mg} / \mathrm{kg}$ buprenorphine twice per day) were given if warranted to eliminate postoperative pain. Note that enucleation results in degeneration of the optic nerve, preventing any subsequent activation of the peripheral visual pathway by light or optic nerve stimulation.

Preparation for adult electrophysiology. Animals were prepared for electrophysiology experiments as described previously (Mao et al., 2011). The ear canals of each ferret were examined before surgery with an otoscope and cleaned if necessary. Atropine (0.4 mg/kg, s.q.) and doxapram ( $2 \mathrm{mg} / \mathrm{kg}$, s.q.) were given as a preanesthetic to counteract bradycardia and to reduce mucosal secretions. Animals were anesthetized with ketamine $(40 \mathrm{mg} / \mathrm{kg}$, i.m. ) and diazepam $(2 \mathrm{mg} / \mathrm{kg}$, i.m.) during the craniotomy procedure. Dexamethasone $(1 \mathrm{mg} / \mathrm{kg}$, i.m.) was given every $24 \mathrm{~h}$ to prevent cerebral edema. For the recording session, the cephalic or femoral vein was cannulated, and an intravenous solution containing a combination of medetomidine $\left(0.022 \mathrm{mg} \cdot \mathrm{kg}^{-1} \cdot \mathrm{h}^{-1}\right)$ and ketamine $(5$ $\mathrm{mg} \cdot \mathrm{kg}^{-1} \cdot \mathrm{h}^{-1}$ ) in lactated Ringer's solution with $5 \%$ dextrose (Bizley et al., 2005; Bizley and King, 2008) was continuously infused at a rate sufficient to maintain a state of unconsciousness $(2-5 \mathrm{ml} / \mathrm{h})$. Atropine $(0.4 \mathrm{mg} / \mathrm{kg}$, s.q. $)$ was given as necessary to counteract the bradycardia caused by medetomidine. The animal was intubated and artificially ventilated using a small animal ventilator (SAR 830/P ventilator; CWE). Body temperature was maintained at $38^{\circ} \mathrm{C}$ with a heating pad. $\mathrm{EKG}$, respiration rate, muscle tone, withdrawal reflexes, and end-tidal $\mathrm{CO}_{2}$ were monitored and recorded every $30 \mathrm{~min}$ at the beginning of surgery and every hour after surgery. Eyes were kept moist with commercial artificial tears solution and protected with custom plano contact lenses (Conforma). The animal was placed in a stereotaxic device, and an incision was made on the top of the head with a scalpel. The temporal muscles were retracted from the skull, and two burr holes (at coordinates 5.5 anterior and \pm 1.5 lateral) were drilled for optic chiasm recording/ stimulation electrodes. To protect the brain from overheating during the drilling, saline was frequently dripped on the surface. Two tungsten rods with Teflon insulation ( 0.008 bare, 0.011 coated; A-M Systems) were advanced in the two holes to a depth $(8-10 \mathrm{~mm})$ that yielded strong visual responses. These tungsten rods were then connected to a stimulus isolation unit (BAK Electronics). A 0.8 - to 1.0 -cm-diameter craniotomy was made over the left auditory cortex. After removing the skull, the ferret AC [defined as primary auditory cortex (A1) and the anterior auditory field (AAF)] was exposed (see Fig. 1) (Kelly et al., 1986). The dura was removed carefully, and the AC was covered with sterile saline. The skull on the right side was cleaned, and a metal bracket was cemented on the surface to stabilize the head. The right ear bar was then released to allow access to the ear for auditory stimulation.

Acoustic stimuli and optic chiasm stimuli. Acoustic stimuli were generated using TDT System II or TDT System III hardware and software (Tucker Davis Technologies). A calibrated earphone (ER-2 insert earphone; Etymotic Research) was placed in the pinna at the entrance to the right ear canal. All auditory stimuli were presented contralateral to the recording site. White noise bursts (5 ms ramp, 40-100 ms duration, 80 $\mathrm{dB}$ SPL) were used to search for sound-responsive units. Pure tones of different frequencies were presented in random order after a responsive neuron was found. Calibrated, pure tones ranging in $2 \mathrm{kHz}$ steps from 2 to $18 \mathrm{kHz}$ or ranging in half octave steps from $500 \mathrm{~Hz}$ to $16 \mathrm{kHz}$ with an intensity of $30-80 \mathrm{~dB}$ SPL ( $50 \mathrm{~ms}$ duration, $5 \mathrm{~ms}$ ramp) were given to define tuning curves. Electrical stimulation of the optic chiasm was used to activate visual fibers. Because visual inputs to the medial geniculate nucleus (MGN) in XM animals are weak and are comprised of W retinal ganglion cells (Sur et al., 1988; Roe et al., 1993; Pallas and Sur, 1994), it was rarely possible to activate visual and multisensory neurons by light. Bipolar electrical stimulation of the optic chiasm was applied (single pulses at $0.5-1 \mathrm{~mA}, 60 \mu \mathrm{s}$ duration) to test whether the units were bimodal.

Extracellular recording. Recordings were made with glass-coated tungsten microelectrodes (1-2 M $\Omega$; FHC). The cortical surface was photographed to record the penetration locations. Penetration sites were chosen to sample randomly while avoiding sulci and blood vessels. The electrode was advanced in $5 \mu \mathrm{m}$ steps down to $2000 \mu \mathrm{m}$ under the pial surface by a hydraulic microdrive (David Kopf Instruments). For each penetration, the first stable unit encountered was isolated and characterized. No more than two recording sites were made in each penetration, although generally two units could be separately isolated at each site using the spike isolation software. Most units were isolated within 800 $\mu \mathrm{m}$ of the pial surface. Sampling densities and locations were comparable across groups. In this research, recording penetrations were limited to A1 and AAF (Bizley et al., 2005). There is a low-frequency reversal at the ventral edge of the primary auditory areas (Bizley et al., 2005, 2008), and recording was stopped when the reversal was encountered (see Fig. 1).

Neural responses were bandpass filtered $(500 \mathrm{~Hz}$ to $5 \mathrm{kHz}$ ), amplified (10,000 times; BAK Electronics), and monitored on a digital oscilloscope (Hameg Instruments). Responses to 5-10 stimulus presentations were gathered from each recording site and digitized at $25 \mathrm{kHz}$. Spontaneous activity was recorded for $50 \mathrm{~ms}$ before each trial to normalize the evoked response levels. Digitized data were acquired by Brainware software (Tucker Davis Technologies). The recording continued for 1-2 d, after which the animal was deeply anesthetized for perfusion and the brain was extracted for histological examination.

Electrophysiological data analysis. Brainware software (Tucker Davis Technologies) was used for offline spike sorting. Biphasic action potentials were extracted by artifact rejection set in Brainware. Single units were isolated according to their waveform, amplitude, and width. Poststimulus time histograms (PSTHs) of the selected single units were gen- 
erated using the same software package. Response latencies were determined by the time between stimulus presentation and the time of the first bin in the PSTH that reached $\geq 20 \%$ above background firing rate. For isolated single units, a frequency response area was reconstructed by summing the responses to each pure tone across 5-10 trials. The boundaries of the frequency tuning curve were defined by the stimuli that yielded excitatory responses above the mean spontaneous activity plus $20 \%$ of the peak rate (Sutter and Schreiner, 1991; Bizley et al., 2005) and plotted using a MATLAB program kindly provided by Prof. Jennifer Bizley (Ear Institute, University College London, London, UK). The response threshold was defined as the lowest sound level that evoked auditory responses. The best frequency (BF) of each unit was defined as the frequency at which the lowest threshold responses were elicited. Some neurons responded similarly to two contiguous frequencies, in which case their BF was defined as the mean of those two frequencies. If neurons responded at a low threshold to two contiguous frequencies, BF was defined as the frequency that induced the larger response. Neurons were defined as tuned if they had single-peaked or double-peaked tuning curves. Neurons that responded to at least three frequencies at the same threshold level were defined as untuned neurons. Bandwidth was determined by the width of the tuning curve at $10 \mathrm{~dB}$ above this minimum threshold. Multisensory units were defined as either neurons that responded to both visual and auditory stimuli or neurons that only responded to one modality but could be significantly modulated by stimulation with the other modality (Stein and Meredith, 1993). Statistical significance was determined by comparing the number of spikes per sweep (counted from PSTHs) as a response to the different stimulus modalities using Student's $t$ test $(p<0.05)$. For calculating the spatial distribution of frequency-tuned neurons, the area of each AC was normalized to a standard circle with a radius of 1 (Fig. 1) (Mao et al., 2011). The locations of recorded units were reconstructed on this normalized AC.

Electrophysiological data were statistically analyzed using SigmaStat software (Systat Software) and PASW statistic 18 (SPSS) and plotted using Sigmaplot (Systat Software) or MATLAB (MathWorks). A one-way ANOVA was used for multiple comparisons of normally distributed data. A one-way ANOVA on ranks (Kruskal-Wallis test) was used for multiple comparisons of non-normally distributed data. A Student's $t$ test was used for normally distributed data. For two group comparison of ranks on nonnormally distributed data, a Mann-Whitney $U$ test was used. Means \pm SEM are given throughout.

Assessment of lesion size. After electrophysiological recordings were completed, animals were deeply anesthetized with sodium pentobarbital $(65 \mathrm{mg} / \mathrm{kg}$, i.p.) and perfused with PBS, followed by $4 \%$ paraformaldehyde in $0.1 \mathrm{M}$ phosphate buffer (PB). Brains were extracted and postfixed in $4 \%$ paraformaldehyde in $0.1 \mathrm{M} \mathrm{PB}$ for $24 \mathrm{~h}$ at $4^{\circ} \mathrm{C}$. The brains were transferred to $30 \%$ sucrose in $0.1 \mathrm{M} \mathrm{PB}$ at $4^{\circ} \mathrm{C}$ after postfixation. After the tissue was infiltrated by the sucrose solution, it was sectioned frozen at 50 $\mu \mathrm{m}$ in the coronal plane for reconstruction of lesions. A series of sections at $200 \mu \mathrm{m}$ intervals was mounted on gelatin-subbed slides and stained for Nissl substance with cresyl echt violet. The volumes of the residual auditory midbrain (ICc) and the residual visual midbrain (sSC) were measured from Nissl-stained sections with a Zeiss microscope using Zeiss Axio Vision 3.1 software (Carl Zeiss). The borders of ICc and sSC were very clear on our Nissl-stained sections (Mao et al., 2011). The volumes of sSC and ICc were calculated as the sum of the size of each measured area multiplied by the length of the interval between assayed sections of $200 \mu \mathrm{m}$ (Fig. 2). Proportions of residual midbrain volume in the lesioned animals were calculated by comparison with an average midbrain volume derived from five normal animals (Mao et al., 2011).

\section{Results}

The goal of this study was to investigate the mechanism through which the XM plasticity that results from neonatal, partial deafferentation of auditory thalamus impairs eventual recovery of auditory function. It is well accepted that AC is subject to unimodal, experience-dependent modification (Zhang et al., 2001, 2002; Insanally et al., 2009; Popescu and Polley, 2010), but how it might be negatively or positively affected by XM modification is less clear. The XM group had neonatal damage to auditory and visual midbrain, redirecting retinal inputs to auditory thalamus (MGN) and thus providing visual activation of AC (Sur et al., 1988). In a previous study, we showed that, after recovery from surgery, XMAC can respond to both auditory and visual stimuli (Mao et al., 2011). We were interested in whether that residual auditory function is compromised by invasion of the ectopic visual inputs. Because damage to both auditory and visual midbrain is necessary to induce XM plasticity, it was important to establish whether AC function was impaired by anomalous visual inputs, loss of auditory inputs, or both. To distinguish between 

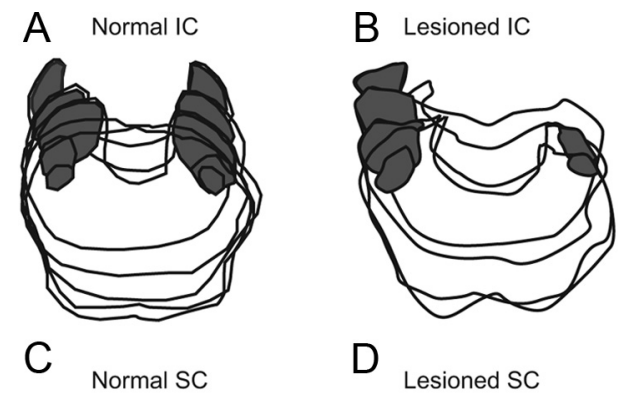

D Lesioned SC
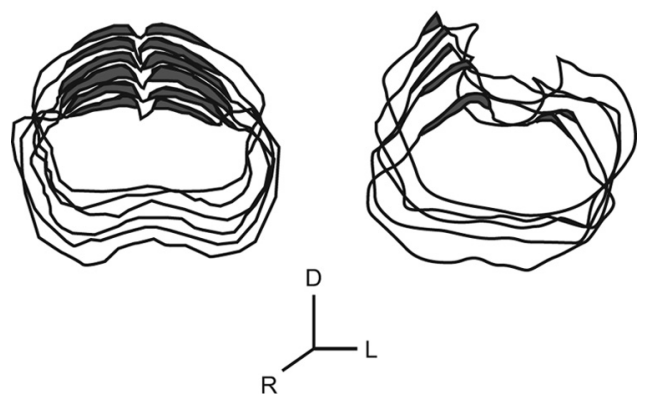

Figure 2. Assessment of midbrain damage. Dark areas represent the $\operatorname{ICC}(\boldsymbol{A}, \boldsymbol{B})$ and $\operatorname{sSC}(\boldsymbol{C}, \boldsymbol{D})$. $A$, An example of a series of sections through the normal IC. $\boldsymbol{B}$, An example of a series of sections through the lesioned IC. $C$, An example of a series of sections through the superficial layers of normal SC. $D$, An example of a series of sections through the superficial layers of lesioned SC. The directional at bottom indicates dorsal (D), rostral (R), and left (L). The left side was oriented on the right in this figure to show rostral sections of ICC in the front after sectioning from caudal to rostral. In all panels, several sections at $400 \mu \mathrm{m}$ intervals are overlain.

these two possibilities, we designed a blind-lesioned group that had the same neonatal midbrain lesions as XM animals but no visual inputs, as a control for the effects of the visual activation of XMAC. Because this group introduces enucleation as another experimental variable, to rule out the effect of enucleation on auditory function, we added a group of blind-only animals. The study thus contained four groups: normal, XM, blind-lesioned, and blind animals.

Twenty-six ferrets in total were used. Eight were in the normal group, nine were in the XM group, six were in the blind-lesioned group, and three were in the blind group. XM ferrets were lesioned on the day of birth (P1). Blind ferrets were enucleated on P1-P7. Enucleation on P7 increased the survival rate of kits and would be expected to have the same effect as enucleation on P1 because the thalamocortical projection does not reach cortex until P14 (Herrmann et al., 1994). Blind-lesioned ferrets were enucleated and lesioned on the same day (P1) to avoid multiple survival surgeries. Statistical analyses included both parametric and nonparametric methods depending on the normality of the sample distribution, but in all cases, we present error bars as SEM.

The residual midbrain volume of XM animals was not significantly different from the residual midbrain volume of blind-lesioned animals

The blind-lesioned animals were introduced as a control for the alternative hypothesis that the changes in XMAC are caused by auditory deafferentation rather than visual activation. Because variable lesion sizes between groups could potentially affect the interpretation of results, we examined whether the lesions in these two groups were similar in size. We compared the volumes of residual midbrain components from these two groups against midbrain volumes from a reference set of normal animals (Mao et al., 2011). We found that the volumes of residual left midbrain (sSC plus ICc) were not significantly different between the XM

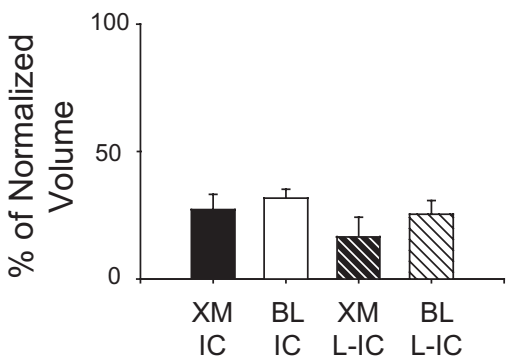

Figure 3. Statistical comparison of residual volumes of $\mathrm{IC}$ in XM and blind-lesioned (BL) animals. The solid bars represent volumes of the left and right colliculi combined, and the striped bars represent volumes of the ipsilesional, left inferior colliculi (L-IC). Error bars show \pm SEM.

Table 1. The proportion of low-frequency neurons and residual midbrain volumes in lesioned animals

\begin{tabular}{|c|c|c|c|c|c|c|}
\hline & $\begin{array}{l}\text { Low-frequency } \\
\text { neurons (\%) }\end{array}$ & L-IC (\%) & R-IC (\%) & $\begin{array}{l}\text { Average } \\
\mathrm{IC}(\%)\end{array}$ & L-SC (\%) & $\begin{array}{l}\text { Average left } \\
\text { midbrain (\%) }\end{array}$ \\
\hline \multicolumn{7}{|l|}{$X M$} \\
\hline $08-240$ & 12.5 & 0 & 12.66 & 6.33 & 38.19 & 19.10 \\
\hline $09-21$ & 0 & 0 & 37.70 & 18.85 & 6.26 & 3.13 \\
\hline 08-201 & 0 & 0 & 34.69 & 17.34 & 5.44 & 2.72 \\
\hline $08-253$ & 0 & 1.06 & 15.47 & 8.26 & 51.12 & 26.09 \\
\hline 09-191 & 3.86 & 5.62 & 23.59 & 14.61 & 11.48 & 8.55 \\
\hline $09-15$ & 0 & 11.00 & 73.36 & 42.18 & 64.36 & 37.68 \\
\hline $11-01$ & 12.5 & 35.96 & 46.29 & 41.12 & 19.84 & 27.90 \\
\hline $09-203$ & 9.76 & 47.86 & 66.59 & 57.23 & 62.90 & 55.38 \\
\hline $09-172$ & 33.33 & 51.38 & 26.95 & 39.17 & 23.79 & 37.58 \\
\hline Average & & $24 \pm 5.9$ & & $27 \pm 6.0$ & & $17 \pm 7.2$ \\
\hline \multicolumn{7}{|c|}{ Blind-lesioned } \\
\hline $10-119$ & 12.5 & 10.41 & 64.53 & 37.47 & 13.69 & 12.05 \\
\hline $11-36$ & 8.70 & 15.90 & 30.07 & 22.99 & 11.87 & 13.89 \\
\hline $10-104$ & 16.67 & 20.28 & 47.83 & 34.05 & 19.00 & 19.64 \\
\hline $10-98$ & 45 & 29.20 & 11.10 & 20.15 & 39.98 & 34.59 \\
\hline $10-110$ & 47.37 & 29.20 & 37.74 & 33.47 & 16.30 & 22.75 \\
\hline $10-116$ & 8.7 & 47.54 & 37.01 & 42.27 & 10.50 & 29.02 \\
\hline Average & & $22 \pm 3.6$ & & $32 \pm 3.5$ & & $25 \pm 5.4$ \\
\hline
\end{tabular}

The midbrain volumes were normalized to average midbrain volumes of normal animals. L, Left; $R$, right.

$(24 \pm 5.9 \%, n=9)$ and blind-lesioned $(22 \pm 3.6 \%, n=6 ; p=$ $0.78, t$ test) groups. In particular, the average volume of residual IC in XM animals was not significantly different from that of the blind-lesioned animals (XM, $27 \pm 6.0 \%, n=9$; blind-lesioned, $32 \pm 3.5 \%, n=6 ; p=0.58$, $t$ test) (Fig. 3, Table 1). Because the majority of projections to left AC come from ipsilateral IC via the left MGN, we also compared the residual volume of left IC between the XM and the blind-lesioned groups. We did not find a significant difference between these two groups (XM, $17 \pm 7.2 \%$, $n=9$; blind-lesioned, $25 \pm 5.4 \%, n=6 ; p=0.41, t$ test) (Fig. 3 , Table 1). These results show that the extent of neonatal midbrain damage was similar in lesioned animals with or without enucleation. Therefore, it is unlikely that different response characteristics in AC can be attributed to differences in neonatal lesion size in the XM compared with the blind-lesioned animals.

\section{Auditory and multisensory neurons in AC of XM animals were tuned to pure tones}

The majority of sound-responsive neurons in the primary auditory areas of normal adult ferrets have a single BF between 1 and $18 \mathrm{kHz}$ (Kelly et al., 1986; Phillips et al., 1988). To test the hypothesis that auditory function in XMAC is impaired by invasion of visual inputs, we first asked whether neurons in XMAC exhibit normal tuning to pure tones. Neurons that had a single peak in 
A

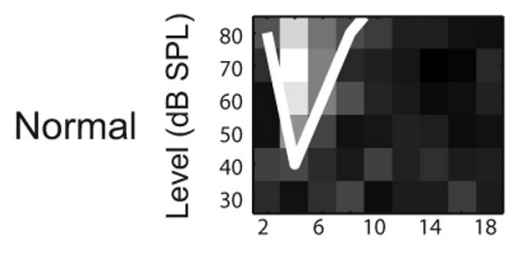

Frequency $(\mathrm{kHz})$

B

Blind

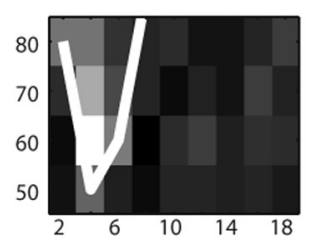

C

BlindLesioned
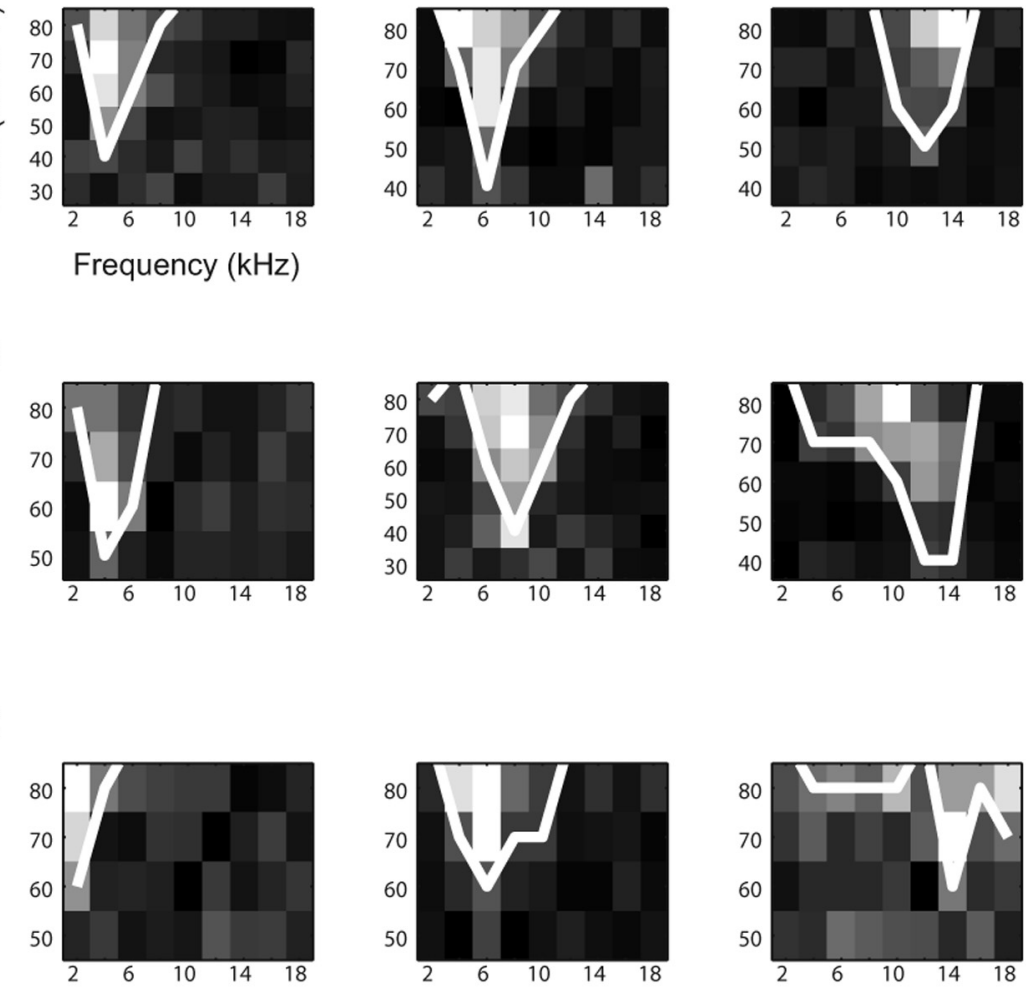

High
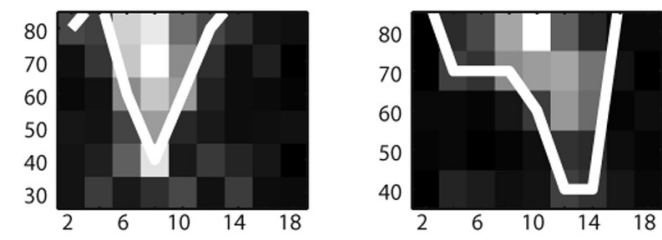

D

\section{Auditory-} XM
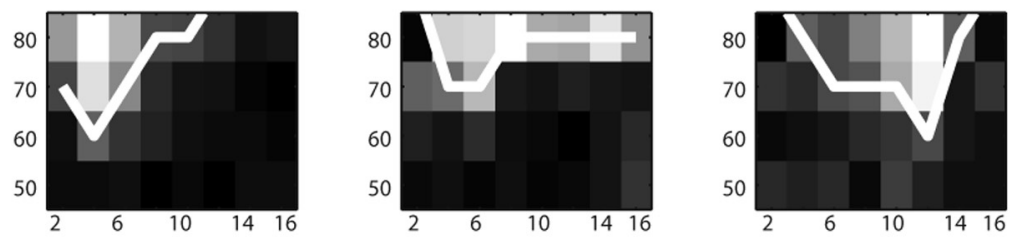

\section{E}

\section{Multisensory- XM}
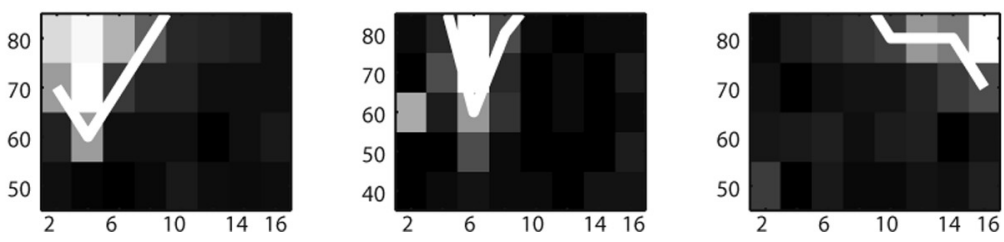

Figure 4. Examples of low-, mid-, and high-frequency tuning curves of auditory and multisensory neurons. $A$, Tuning curves of auditory neurons in normal AC. $B$, Tuning curves of auditory neurons in the $A C$ of blind animals. C, Tuning curves of auditory neurons in the AC of blind-lesioned animals. D, Tuning curves of auditory neurons in the XMAC. $E$, Tuning curves of multisensory neurons in the XMAC. $F$, An example of a tuning curve from a dual-peak auditory neuron. Dual-peak auditory neurons have two BFs. $G$, An example of a tuning curve from a no-BF auditory neuron (No BF). No-BF auditory neurons respond to multiple pure tones but do not show any preference.

their sound frequency tuning curves were considered to be tuned to a single BF (for details, see Materials and Methods). Neurons that responded to frequencies $\geq 12 \mathrm{kHz}$ were defined as highfrequency neurons, neurons that responded to frequencies between $\geq 6 \mathrm{kHz}$ and $<12 \mathrm{kHz}$ were defined as mid-frequency neurons, and neurons that responded to frequencies $<6 \mathrm{kHz}$ were defined as low-frequency neurons. We found that nearly all of the recorded neurons from normal, blind, blind-lesioned, and
$\mathrm{XM}$ ferrets were tuned to pure tones in these ranges (Fig. 4). This was true of auditory-only neurons in XMAC (Aud-XM neurons) and of multisensory neurons in XMAC (Multi-XM neurons). Thus, neurons in XMAC and AC of blind-lesioned animals retain selectivity for sound frequency despite the significant early damage to the auditory midbrain (IC).

In addition to the single-peak neurons, we encountered a small proportion of multipeaked auditory neurons in the le- 

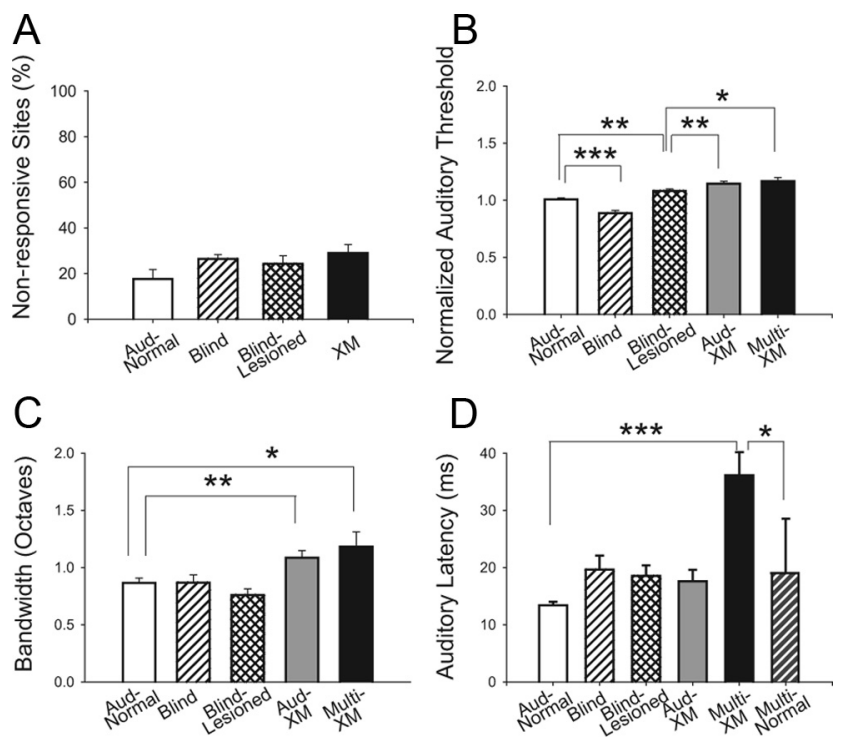

Figure 5. The response characteristics of neurons in XM and control animals. $\boldsymbol{A}$, The proportion of nonresponsive recording sites across groups. No significant difference was found despite comparable sampling densities and locations. $\boldsymbol{B}$, Normalized thresholds of auditory responses across groups. $\boldsymbol{C}$, Bandwidth of auditory responses across groups. $\boldsymbol{D}$, Latencies of auditory responses across groups. Error bars show \pm SEM. ${ }^{*} p<0.05,{ }^{* *} p<0.01,{ }^{* * *} p<0.001$.

sioned animals only (XM group, 3.4\%, $n=8$ from 235 neurons; blind-lesioned group, 12\%, $n=18$ from 152 neurons) (Fig. $4 F$ ). These multipeaked neurons had two BFs, either at 4 and $12 \mathrm{kHz}$ or at 8 and $16 \mathrm{kHz}$, with 1 to 2 octaves between peaks, distinct from typical multipeaked neurons that have been described in normal auditory cortex (Sutter and Schreiner, 1991). The proportion of auditory neurons that were not tuned to pure tones was $2 \%$ for the normal group ( $n=4$ from 192 neurons), $1 \%$ for the XM group ( $n=2$ from 235 neurons), $1.3 \%$ for the blindlesioned group ( $n=2$ from 152 neurons), and $3.3 \%$ for the blind group ( $n=3$ from 89 neurons) (Fig. $4 G$ ).

\section{Visual inputs affect the threshold of auditory and multisensory cortical neurons to sound}

We also examined whether the ectopic visual inputs reduced the responsiveness of XMAC neurons to sound. We did not find any significant differences between groups in the proportion of nonresponsive recording sites (Fig. 5A). For the responsive sites, because our intention was to compare the differences in auditory response thresholds between groups rather than determine absolute threshold values, we normalized all auditory threshold measurements to the average threshold of the set of normal auditory neurons, which was $51 \pm 0.7 \mathrm{~dB}$ SPL $(n=154)$. We found significant differences in thresholds of sound-responsive neurons between the normal, blind, blind-lesioned, and XM groups (ANOVA, $p<0.001$; Fig. 5B). First, the normalized thresholds of auditory neurons in blind-lesioned animals were significantly higher than those of normal auditory neurons (normal, $1.01 \pm$ $0.015, n=154$; blind-lesioned, $1.08 \pm 0.016, n=108 ; p<0.001$, Fisher's least significant difference (LSD) test). These results suggest that the sensitivity to sound in the $\mathrm{AC}$ was impaired by IC lesion, as would be expected. Second, we found that the thresholds in XMAC were significantly higher than those in the AC of blind-lesioned animals (Aud-XM neurons, $1.15 \pm 0.021, n=$ 121; Multi-XM neurons, $1.17 \pm 0.03, n=22$; Aud-XM vs blindlesioned, Multi-XM vs blind-lesioned, $p<0.05$, Fisher's LSD test). These findings suggest that the ectopic visual invasion of
XMAC further decreases auditory sensitivity, in addition to the impairment brought by IC lesion. Third, we did not find any significant difference in normalized thresholds between the multisensory neurons and the auditory neurons in XMAC (Aud-XM vs Multi-XM, Fisher's LSD test, $p=0.6$ ). Finally, the normalized thresholds of auditory neurons in blind animals were significantly lower than those of normal auditory neurons (blind, $0.89 \pm 0.023, n=84, p<0.001$, Fisher's LSD test). These data imply that neonatal visual deafferentation increases the sensitivity of auditory neurons to sound, a finding that has been reported by others in several model systems (see Discussion).

\section{Auditory neurons in AC of XM animals had broader tuning to sound frequency than auditory neurons in normal AC}

The width of the tuning curves of sound-responsive neurons has been used as an important metric for the sensitivity of auditory responses (for review, see Aitkin et al., 1984). We expected that auditory deafferentation would result in broader tuning of auditory responses in the AC. In support of this prediction, we found that the bandwidths of sound-responsive neurons were significantly different between normal, blind, blind-lesioned, and XM animal groups (Aud-XM and Multi-XM groups, Kruskal-Wallis test, $p<0.05$ ) (Fig. 5C). On the one hand, we found that the bandwidths of auditory and multisensory neurons in XMAC were significantly broader than those of auditory and multisensory neurons in normal animals (normal, $0.87 \pm 0.04, n=154$; Aud-XM, $1.09 \pm 0.06, n=121$; normal vs Aud-XM, $p=0.008$, Mann-Whitney $U$ test; Multi-XM, $1.18 \pm 0.13, n=22$; normal vs Multi-XM, $p<0.05$, Mann-Whitney $U$ test). These data suggest that visual invasion of XMAC decreases the sharpness of auditory tuning. On the other hand, we did not find any significant differences in sharpness of tuning between the normal group and the two control groups (blind and blind-lesioned groups) (blind, $0.87 \pm 0.07, n=84$; blind-lesioned, $0.76 \pm 0.05, n=108$; normal vs blind and normal vs blind-lesioned, $p>0.05$, Mann-Whitney $U$ test). These data demonstrate that, although auditory cortical neurons in the XM animals have broader tuning, the sharpness of tuning is not affected in the blind-lesioned animals, suggesting that possible circuitry underlying tuning such as thalamocortical convergence or lateral inhibition in adult $\mathrm{AC}$ is not altered by neonatal IC damage. Thus, XM visual input decreases the specificity of auditory responses in XMAC, suggesting that XM plasticity could interfere with auditory perception.

\section{Multisensory neurons in AC of XM animals had a longer latency response to sound stimuli than unisensory auditory neurons in either normal AC or XMAC}

In previous research, we measured response latencies to visual stimuli and found that multisensory neurons in XMAC had longer than normal latencies (Mao et al., 2011). Here we calculated the latencies of auditory responses to gain insight into whether the multisensory neurons obtain their auditory inputs directly from thalamus or cortical sources. We found that the latencies of sound-responsive neurons were significantly different between groups (Kruskal-Wallis test, $p<0.05$ ) (Fig. 5D). Latencies of auditory responses in the multisensory neurons of XMAC were nearly three times longer than those of normal auditory responses (normal, $13 \pm 0.6 \mathrm{~ms}, n=136$; Multi-XM, $36 \pm 10 \mathrm{~ms}, n=22 ; p=0.001$, Mann-Whitney $U$ test), whereas latencies of auditory neurons in XMAC were not significantly different from those of normal auditory neurons (normal, $13 \pm 0.6 \mathrm{~ms}, n=136$; Aud-XM, $18 \pm 2.0 \mathrm{~ms}, n=121 ; p=0.66$, Mann-Whitney $U$ test). These results suggest that auditory neurons in XMAC receive direct thalamocortical projections, whereas multi- 
sensory neurons are more likely to obtain their auditory inputs from other cortical sources. As expected, we did not find significant differences in latency between the normal and the two control groups (blindlesioned, $19 \pm 1.8 \mathrm{~ms}, n=126, p=0.366$, Mann-Whitney $U$ test; blind, $19.6 \pm 2.4 \mathrm{~ms}$, $n=84, p=0.24$, Mann-Whitney $U$ test). For comparison, we also measured the auditory latencies of the small proportion of multisensory neurons seen in normal AC. The auditory latencies of the multisensory neurons in normal AC were not significantly different from those of normal auditory neurons (multi-normal, $19 \pm 9.4, n=$ 10 , Mann-Whitney $U$ test, $p=0.18$ ), supporting the idea that multisensory neurons in normal AC may receive direct projections from thalamus and function in auditory processing as do typical auditory-only neurons.

The tonotopic arrangement of auditory and multisensory neurons was altered by XM plasticity

Tonotopic arrangement is an essential characteristic of neurons in primary auditory areas. We found auditory and visual neurons to be intermingled in XMAC in our previous study (Mao et al., 2011), leading us to hypothesize that invasion of ectopic, XM visual inputs to auditory thalamus disrupts tonotopic order. To examine whether IC lesion affects the distribution and proportion of neurons tuned to each part of the sound frequency scale, we divided the scale into three bins (low-frequency, $<6 \mathrm{kHz}$; midfrequency, $6-12 \mathrm{kHz}$; high-frequency, $>12 \mathrm{kHz}$ ) and plotted the topographic locations of units responding in these ranges.

\section{The tonotopic map of auditory neurons in normal AC}

We first mapped the spatial distribution of low-, mid-, and highfrequency neurons across the primary auditory areas in normal animals, using in vivo extracellular recording, for comparison with the other groups. Figure 6 shows examples of the electrode penetration locations and neuronal response types from recordings in normal AC. In each case, the circles represent a single responsive unit. Generally, we could isolate two neurons from each penetration using offline spike sorting. As reported in previous research (Kelly et al., 1986; Phillips et al., 1988), we found that the tonotopic map of auditory neurons in normal ferrets showed a high- to low-frequency trend approximately along the mediolateral axis of AC.

\section{Tonotopic arrangement of auditory neurons in XMAC}

To determine whether XM visual inputs can disrupt topography of auditory neurons, we plotted the tonotopic maps in the AC of XM cases. We found that high-, mid-, and low-frequency auditory neurons in XMAC were scattered rather than being arrayed along the mediolateral axis as observed in normal animals (Fig. 7 ). These results suggest that auditory tonotopy was severely disrupted by the visual invasion into AC of XM animals.
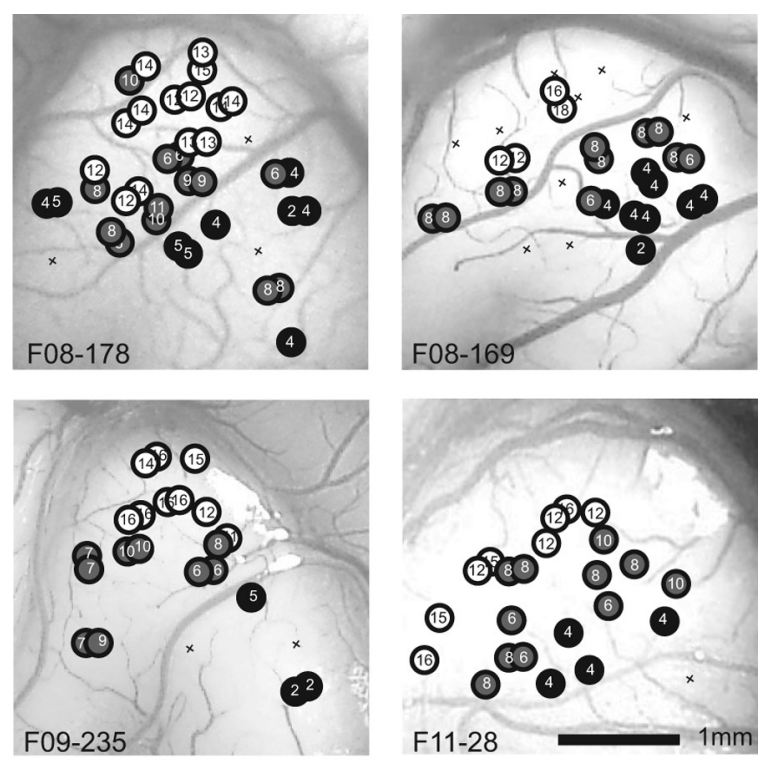

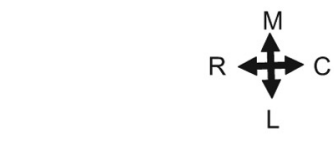

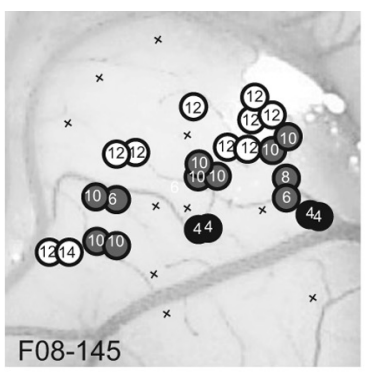

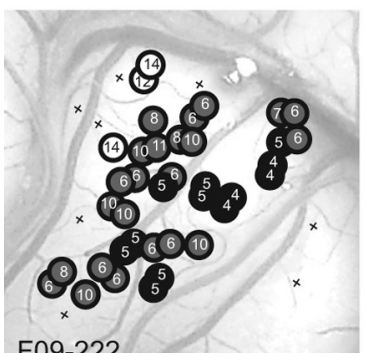

F09-235

Reconstruction of locations of frequency-tuned neurons in normal AC. Each panel exhibits data from one animal ( 6 of examples are shown). Each circle represents one auditory unit, and the numbers in the center indicate BFs, expressed in kilohertz. ind to sound $\geq 6 \mathrm{kHz}$ and $<12 \mathrm{kHz}$ ). Dark circles represent low-frequency neurons (responding to sound $<6 \mathrm{kHz}$ ). X indicates a non-responsive site. Scale bar, $1 \mathrm{~mm}$. R, Rostral; C, Caudal; M, medial; L, lateral.

\section{Tonotopic arrangement of multisensory neurons in XMAC}

In addition to assaying the tonotopic arrangement of auditory neurons, we also reconstructed the tonotopic map of multisensory neurons in XMAC (Fig. 8). The sample size of multisensory neurons was smaller than that of auditory neurons in XMAC because they were rarely encountered. In some cases (Fig. $8 B, C$ ), we recorded only high- and mid-frequency neurons. In others (Fig. $8 A, F$ ), the majority of recorded multisensory neurons were tuned to mid- and low-frequency sounds. Quantified distributions of frequency-tuned neurons in pooled data from this group are compared with those of the normal group below.

\section{Tonotopic arrangement of auditory neurons in AC of blind-lesioned animals}

To test the hypothesis that the ectopic visual inputs and not the deafferentation of MGN are responsible for disrupting the tonotopic map in the XMAC, we recorded neurons from AC of the blind-lesioned group. These cases had deafferentation of MGN but no retinal input and thus no visual activation of AC. In support of our hypothesis, we found that the tonotopy of AC in the blind-lesioned animals was normal. Neurons responding from high to low BFs were aligned along the mediolateral axis as in normal AC (Fig. 9). These data argue that neonatal IC damage (MGN deafferentation) alone did not disrupt tonotopy of auditory neurons in AC of the blind-lesioned animals.

\section{Tonotopic arrangement of auditory neurons in AC of blind animals}

We also examined the effect of binocular enucleation alone by mapping the distributions of auditory neurons in AC of the blind animals 
A

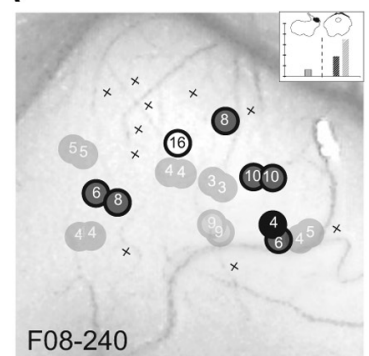

D

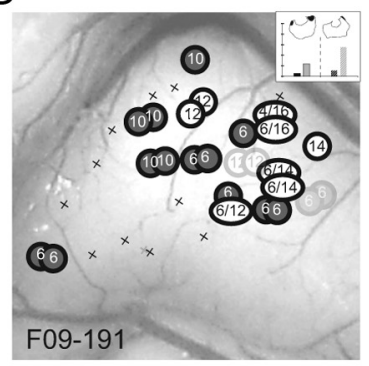

High-frequency neuron

Mid-frequency neuron

Low-frequency neuron

$\times \quad$ Non-responsive penetration
B

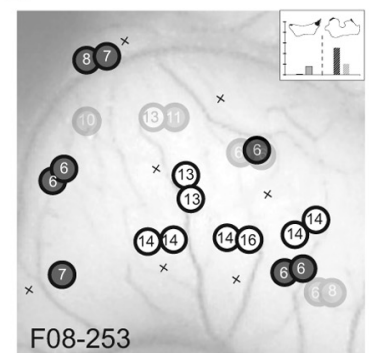

$\mathrm{E}$

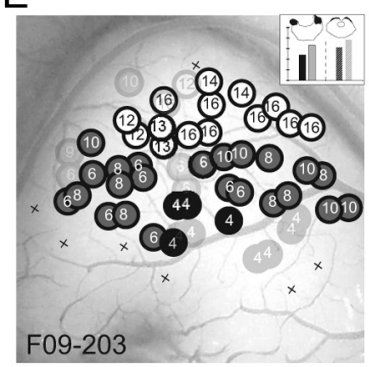

C

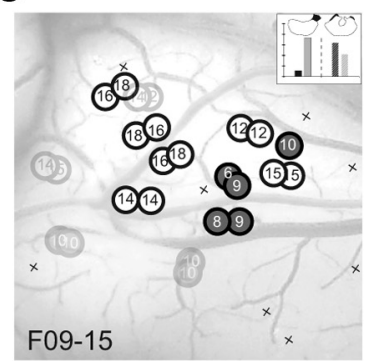

F

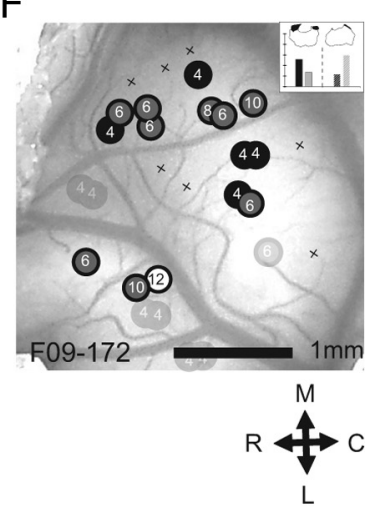

Each number within a circle represents the best frequency (BF) of each neuron

Figure 7. Reconstruction of locations of frequency-tuned auditory neurons in AC of XM animals. Semitransparent circles represent multisensory neurons that were recorded at the same time. Each panel shows data from one animal ( 6 of 9 examples are shown). $\boldsymbol{A} \boldsymbol{- F}$, The panels are ordered from small residual left IC to large residual left IC. The insets show the volumes of residual midbrain from each animal. The $y$-axis represents the proportion of spared midbrain as a percentage of the average volume of normal midbrains. The maximum is therefore $100 \%$. Above the insets to the left is a section of $\mathrm{IC}$ and on the right is a section of $\mathrm{SC}$ from the same animal. The dark areas in these insets represent the ICC and the $\mathrm{SSC}$. Corresponding to these two figures, the four histogram bars on the $x$-axis represent the residual volumes of left IC, right IC, left SC, and right SC, from left to right. $R$, Rostral; $C$, Caudal; M, medial; L, lateral.

in which the midbrain was intact, to rule out the alternative hypothesis that removal of the eyes was somehow responsible for the normal tonotopy in the blind-lesioned group. Figure 10 shows the penetration locations and the neuronal response types from three cases. We found that the tonotopic maps in AC of blind animals were arranged with high to low BFs along the mediolateral axis, as in normal tonotopic maps.

\section{Quantitative comparison of tonotopic maps between groups}

As is typical in long-term recording situations, there was some variation between animals in the number of neurons isolated and recorded. To reduce the effect of this variability on the data analysis, the recorded primary auditory areas were normalized with respect to their area and were fit to a circle that standardized their orientation with respect to the posterior suprasylvian sulcus and the pseudosylvian sulcus, as in our previous study (Mao et al., 2011) (Fig. 11; see also Fig. 1). Although the tonotopic map in ferrets is oriented roughly along the mediolateral axis, it is important to consider that the exact orientation of tonotopic maps varies between ferrets (Kelly et al., 1986; Phillips et al., 1988), as can be seen in Figure 6. Therefore, the combined plots are useful for comparing between groups but in themselves will blur the map detail somewhat. Our pooled data from normal AC are consistent with the results from previous ferret research, with high frequencies represented medially and low frequencies laterally (Fig. 11A). Penetrations were not skewed by location within each auditory cortex, and thus our collective sampling was

even across different parts of the tonotopic map. Furthermore, the sampling rate was not correlated with the degree of tonotopy, arguing against sampling rate as a biasing factor in our interpretation of the data. The insets in Figure $11 \mathrm{~A}$ and in the other panels show the center of spatial distribution of the units tuned to low-, mid-, and highfrequency stimuli, with error bars showing the SEM of these distributions. In normal $\mathrm{AC}$, there is a mediolateral progression in the spatial distribution centers in each frequency tuning category.

We also plotted the distribution of the group of multisensory neurons recorded in normal AC (Fig. 11B). As we showed in a previous study (Mao et al., 2011), multisensory neurons in normal AC were preferentially located laterally. Consistent with this location in the tonotopic map, most were tuned to low and middle frequencies.

In contrast to the tonotopic arrangement seen in normal AC, auditory neurons in XMAC were distributed diffusely across the entire AC (Fig. 11C). As a result, the distribution centers of the different frequency tuning classes were overlapped (Fig. 11C, inset), further reflecting the lack of tonotopy. Interestingly, the distribution centers of the frequency classes of multisensory neurons in XMAC retained some tonotopic order along the mediolateral axis, with the distribution center of the high-frequency neurons separated from the distribution centers of low- and mid-frequency neurons (see below for quantification). Conversely, the mid- and low-frequency multisensory neurons were more diffusely distributed in XMAC (Fig. 11D). The long latencies of Multi-XM neurons (compare with Fig. 5C) suggest that they receive indirect corticocortical inputs rather than direct thalamocortical inputs. Together, these results suggest that visual invasion may affect the thalamocortical pathway more severely. However, we cannot rule out the possibility that the nonoverlapping location of high-frequency neurons results from sampling error attributable to the low proportion of high-frequency neurons in this group ( 8 of 61 ; Fig. $11 D$ ).

The tonotopic organization of auditory neurons in AC of blind-lesioned animals and blind animals was very similar to the tonotopy in the normal group (Fig. $11 E, F$ ). The tuning progressed from high to low along the mediolateral axis, as reflected by the distribution centers seen in the insets.

Overall, these results suggest that the ectopic visual inputs led to a disrupted topography of frequency-tuned neurons in XMAC. In combination with the higher thresholds and broader tuning, it is clear that auditory function of XMAC is compromised. The tonotopic organization of AC in each group will be analyzed and discussed below.

\section{The spatial distribution of high- and low-frequency neurons were altered in XM animals}

Because the tonotopic map in normal ferret $\mathrm{AC}$ is oriented along the mediolateral axis, we quantified the mediolateral location of frequency-tuned neurons across groups (Fig. 12, smaller numbers 
represent more lateral locations). We found that high-frequency auditory neurons in XMAC were located significantly more laterally than those in normal AC $(p<0.001$, ANOVA; Fig. 12A,D), whereas lowfrequency auditory neurons in XMAC were located significantly more medially than those in normal AC $(p<0.05, t$ test; Fig. $12 C, D)$. No significant difference was found with respect to the location of midfrequency neurons across the five groups $(p>0.05, t$ test; Fig. 12B,D). These data suggest that high- and low-frequency neurons are more overlapped in XMAC than in normal AC, as suggested by the insets in Figure $11 C$. We did not observe any significant difference between normal and blindlesioned groups or normal and blind groups $(p>0.05, t$ test; Fig. 12A-C), suggesting that in the blind-lesioned animals the auditory pathway can compensate for early damage to IC and contribute normally to auditory tonotopy in AC. Because the enucleation did not affect tonotopy in ferret AC, the data imply that the disruption of tonotopy in the XM group was caused by the ectopic visual invasion.

\section{The frequency distribution of tuned neurons was not altered by XM plasticity}

We showed in a previous study that an increasing degree of damage to IC is associated with a decrease in the proportion of auditory neurons and an increase in the proportion of visual and multisensory neurons in XMAC (Mao et al., 2011). In ferrets and cats, IC is arranged from low to high frequency along its dorsoventral axis (Merzenich and Reid, 1974; Moore et al., 1983). Because we use a dorsal approach to lesion the IC of XM and blind-lesioned ferrets, the dorsal aspect of IC is preferentially damaged. Therefore, we asked in the current study whether all frequencies were represented in XMAC. We hypothesized that low-frequency neurons would be lost in the lesioned ferrets. Alternatively, neonatal, partial damage to IC could trigger a compression of the tonotopic map of the IC, similar to what occurs with neonatal, partial lesions of SC (Finlay et al., 1979; Pallas and Finlay, 1989). In that case, we would expect all frequencies to be represented. We did not find any significant difference in the proportions of mid- and high-frequency neurons between groups ( $p>0.05$, ANOVA; Fig. 13). The proportion of lowfrequency neurons in the Aud-XM group was significantly lower than that in the normal group ( $p=0.005, t$ test; Fig. $13 C$ ), suggesting a failure of the IC tonotopic map to compress. This interpretation does not consider the fact that many of the soundresponsive neurons in XMAC are bisensory, however. When we included all low-frequency-tuned neurons (Aud-XM plus Multi$\mathrm{XM}$ ) in the analysis, we found that the proportion of lowfrequency neurons was not significantly different from that in the normal group ( $p>0.05$, $t$ test; Fig. $13 C$ ). Together, these results suggest that dorsal midbrain lesions do not eliminate lowfrequency-tuned neurons from the pathway between IC and AC. The apparent compression of the tonotopic map in IC is an interesting finding that warrants future study.

Mid-frequency neuron
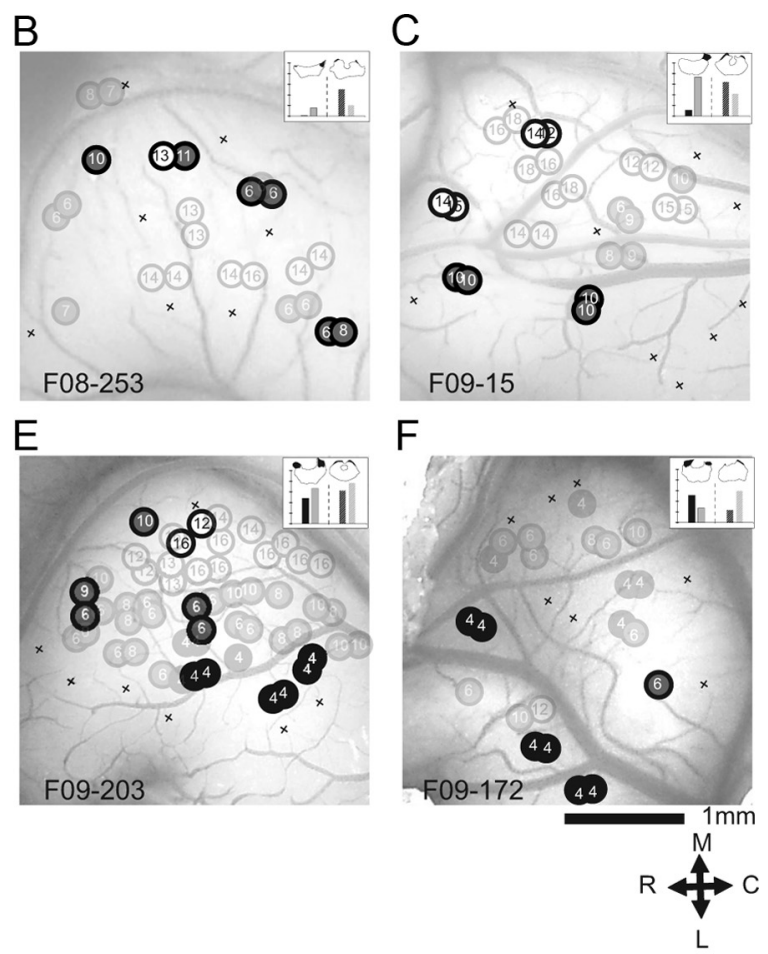

\section{.}

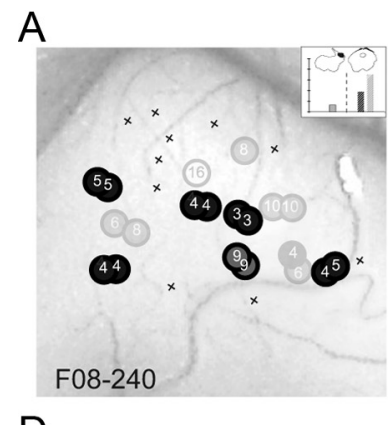

\section{$\mathrm{B}$}

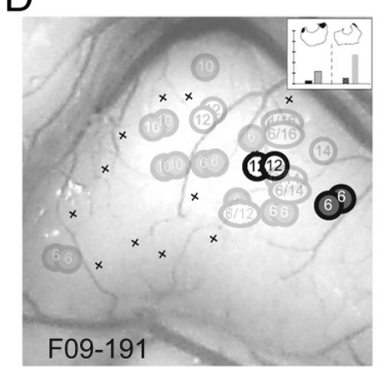

- 203

$x \quad$ Non-responsive penetration

Figure 8. Reconstruction of locations of frequency-tuned multisensory neurons in AC of XM animals. Semitransparent circles represent auditory neurons that were recorded in the same session. Each panel exhibits data from one animal ( 6 of 8 examples are shown). $\boldsymbol{A}-\boldsymbol{F}$, The panels are ordered from small residual left IC to large residual left IC. The insets show the volumes of residual ventions as in Fig. 7).

\section{Discussion}

In a previous study (Mao et al., 2011), we demonstrated that, contrary to previous reports, AC retains its ability to respond to sound stimuli after invasion of XM visual inputs. In the present study, we tested the hypothesis that such XM plasticity negatively affects residual auditory cortical function. We found that considerable auditory function remains in XMAC, in that most neurons respond to sound and have a preferred frequency. However, the sensitivity to sound, the sharpness of tuning, and the organization of the tonotopic map in XMAC are impaired. These results imply that sound discrimination ability is still compromised several months after surgery. The results from the blind-lesioned control group show that it is the anomalous visual input to auditory cortex and not simply deafferentation of the auditory pathway that is responsible for the impairment of auditory function, suggesting that loss of input to auditory thalamus can be compensated for during recovery. In the blind group, the auditory map was normal but the sensitivity of auditory neurons to sound was increased, suggesting that visual impairment can boost auditory processing ability, as seen in blind humans (Röder et al., 1999).

\section{Auditory function is impaired by invasion of ectopic visual inputs}

$\mathrm{XM}$ plasticity has been widely studied in auditory, visual, and somatosensory systems. For example, AC of early-deaf cats is recruited for somatosensory and visual functions (Lomber et al., 2010; Meredith and Lomber, 2011). Auditory prostheses can rescue auditory function to some extent in deaf patients and animal 
A

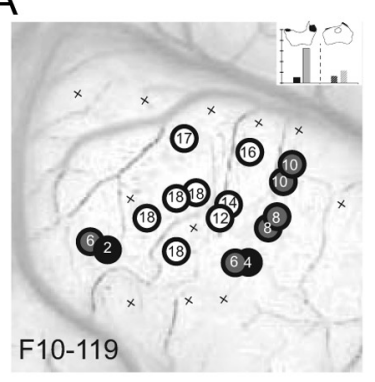

D

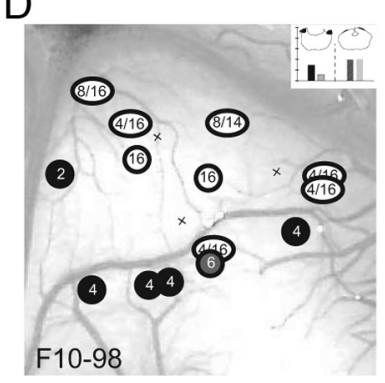

B

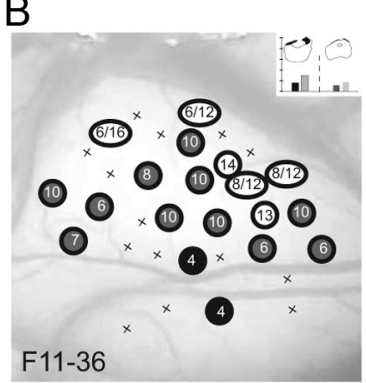

E

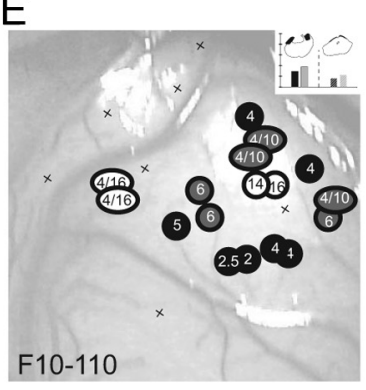

C

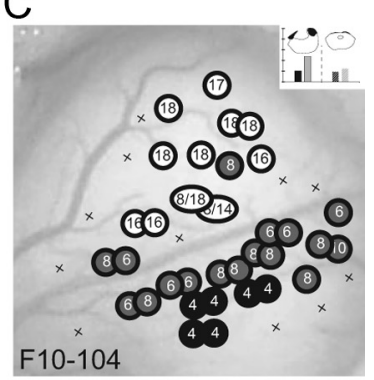

$\mathrm{F}$

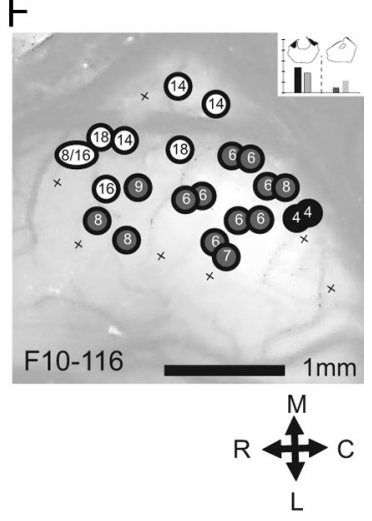

High-frequency neuron

Mid-frequency neuron

Low-frequency neuron

$x \quad$ Non-responsive penetration

Each number within a circle represents the best frequency (BF) of each neuron

Figure 9. Reconstruction of locations of frequency-tuned neurons in AC of blind-lesioned animals. Each panel shows data from one animal (6 of 6 examples are shown). $\boldsymbol{A}-\boldsymbol{F}$, Animals are listed in order from small residual left IC to large residual left IC. Conventions as in Figure 7.
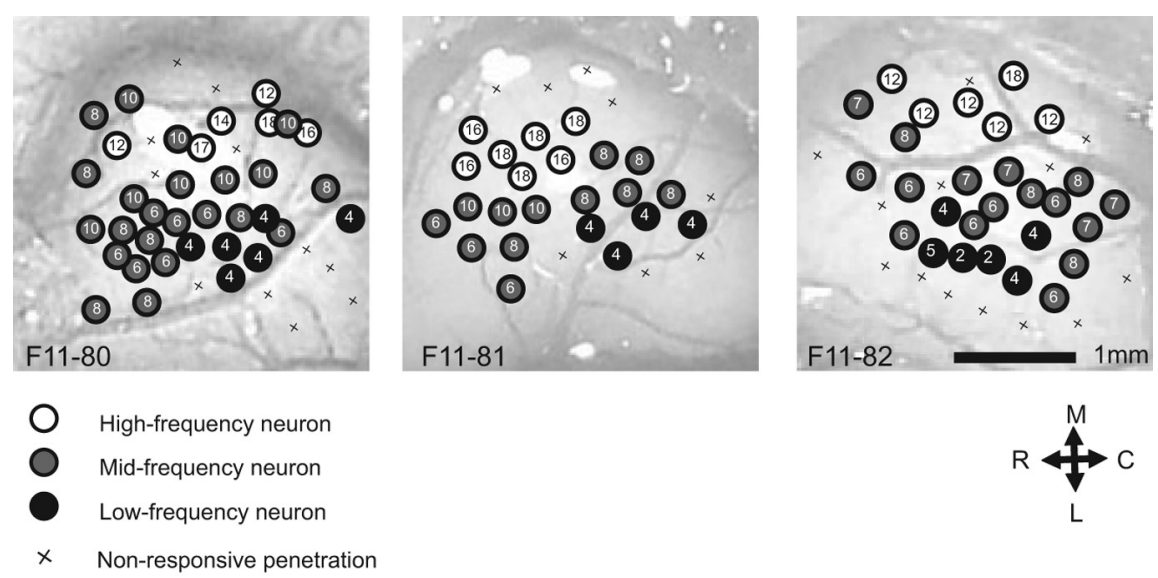

Each number within a circle represents the best frequency (BF) of each neuron.

Figure 10. Reconstruction of locations of frequency-tuned neurons in AC of three blind animals. Conventions as in Figure 7.

models (Klinke et al., 2001; Middlebrooks et al., 2005; Kral, 2007). However, the success of cochlear implants can be compromised if AC exhibits XM activation before implantation is performed (Lee et al., 2001). The basis for this effect is unknown. Here, using a model system in which the degree of XM contamination of AC can be varied experimentally, we addressed whether visual input can negatively impact residual function in the auditory pathway. We found that tonotopy was disrupted by $\mathrm{XM}$ inputs. We also found that response thresholds and sharpness of auditory tuning were degraded in the XMAC group compared with the control groups. These results demonstrate that, after recovery from neonatal midbrain damage, ectopic visual inputs to the auditory pathway impair auditory function in several ways, providing a possible explanation for the deficits remaining after cochlear prostheses are provided to earlydeaf patients. They may also provide insight into the phenomenon of tinnitus that can occur after hearing loss. As in patients who can perceive their amputated limb after surgery, tinnitus patients may hear phantom tones or noise in a quiet environment (Jastreboff, 1990). Tinnitus may be triggered or modulated by XM inputs (Cacace, 2003). We show that many new multisensory neurons are created in $\mathrm{XMAC}$ and that they can respond to optic nerve stimulation while simultaneously being tuned to particular sound frequencies. These novel multisensory neurons provide a possible way to explain how visual activity may express itself as auditory stimulation, creating perceptual confusion and thus phantom sounds. These results suggest avenues for additional study of tinnitus patients with peripheral auditory damage and may also help to guide a general understanding of how maladaptive plasticity can occur after brain damage, interfering with rehabilitation.

\section{Auditory function of multisensory neurons}

In addition to the classically multisensory brain areas, such as the SC and anterior ectosylvian sulcus (Meredith and Stein, 1983; Wallace et al., 1992), primary sensory cortices have been found to exhibit multisensory responses, although to a lesser extent (Schroeder et al., 2003; Wallace et al., 2004; Ghazanfar and Schroeder, 2006; Bizley and King, 2009). Experiments on normal ferrets show that multisensory neurons in A1 have frequency tuning similar to that seen in auditory-only neurons (Bizley et al., 2007). Consistent with these results, we found that bisensory neurons in XMAC exhibited normal auditory characteristics. They were not only tuned to pure tones but were arranged in a tonotopic manner. This frequency selectivity and tonotopic arrangement of multisensory neurons in XMAC suggest that their dominant functions are auditory, although they receive visual input. We also found that multisensory neurons in XMAC had longer response latencies to sound stimuli than multisensory neurons in normal AC. It is possible that auditory neurons receiving direct thalamocortical auditory input are less likely to receive XM visual inputs than are auditory neurons receiving longer-latency auditory inputs. The competition between afferents may push visual afferents to innervate neurons that receive corticocortical auditory connections, giving those neurons longer auditory latencies. 
Multipeaked auditory neurons appear in XM and blind-lesioned animals

Most auditory neurons recorded in normal A1 of ferrets are single peaked with very narrow tuning curves (Kelly et al., 1986; Phillips et al., 1988). We observed some neurons in AC of XM animals and the blind-lesioned animals but not in normal AC, which had more than one BF, creating multiple peaks in their tuning curves. Unlike multipeaked neurons reported by others in normal AC (Sutter and Schreiner, 1991), the two peaks were widespread in frequency, making it unlikely that unmasking of surround inhibition (Rajan, 1998, 2001) could provide an explanation. Alternatively, residual auditory inputs from subcortical auditory nuclei may sprout and project to adjacent areas in MGN or AC. Whatever the explanation, the creation of multipeaked tuning curves in XMAC implies that auditory neurons in XMAC have lost considerable frequency specificity as a result of the loss of some auditory input and gain of visual input. Our finding that tuning curves in XMAC were broader than those in normal AC supports this idea.

\section{Auditory cortical function is primarily} normal in blind-lesioned animals Research on peripheral deafferentation in the somatosensory system (Merzenich et al., 1983; Kaas, 1991), auditory system (Irvine, 2000), and visual system (Lund and Lund, 1976) shows that sensory responsiveness can essentially recover in the denervated areas. For example, in animals with partial cochlear lesions, an area of expanded representation corresponding to the lesion-edge frequencies is found in IC (Harrison et al., 1993, 1998; Irvine et al., 2003), medial geniculate body (Kamke et al., 2003), and AC (Rajan et al., 1993; Kakigi et al., 2000; Irvine et al., 2001). In this study, we ablated the dorsal aspect of the IC of neonatal ferrets (Fig. 2), which may have resulted in loss of sensory drive from low- to mid-frequency channels (Merzenich and Reid, 1974; Moore et al., 1983; Servière et al., 1984). We found that auditory tuning and tonotopy in the blind-lesioned animals were not significantly different from those in normal AC. These results suggest that the tonotopic map is complete but compressed rather than incomplete after recovery from IC damage, as occurs in visual midbrain SC after neonatal damage (Finlay et al., 1979), and thus that the IC has the potential to rearrange its connections when it is damaged. This may have been facilitated by the late onset of hearing (approximately P30 in ferrets) (Moore and Hine, 1992) and the fact that the auditory afferents reach the IC but have not yet segregated into frequency bands at birth when lesions are made (Brunso-Bechtold and Henkel, 2005; Henkel et al., 2007).
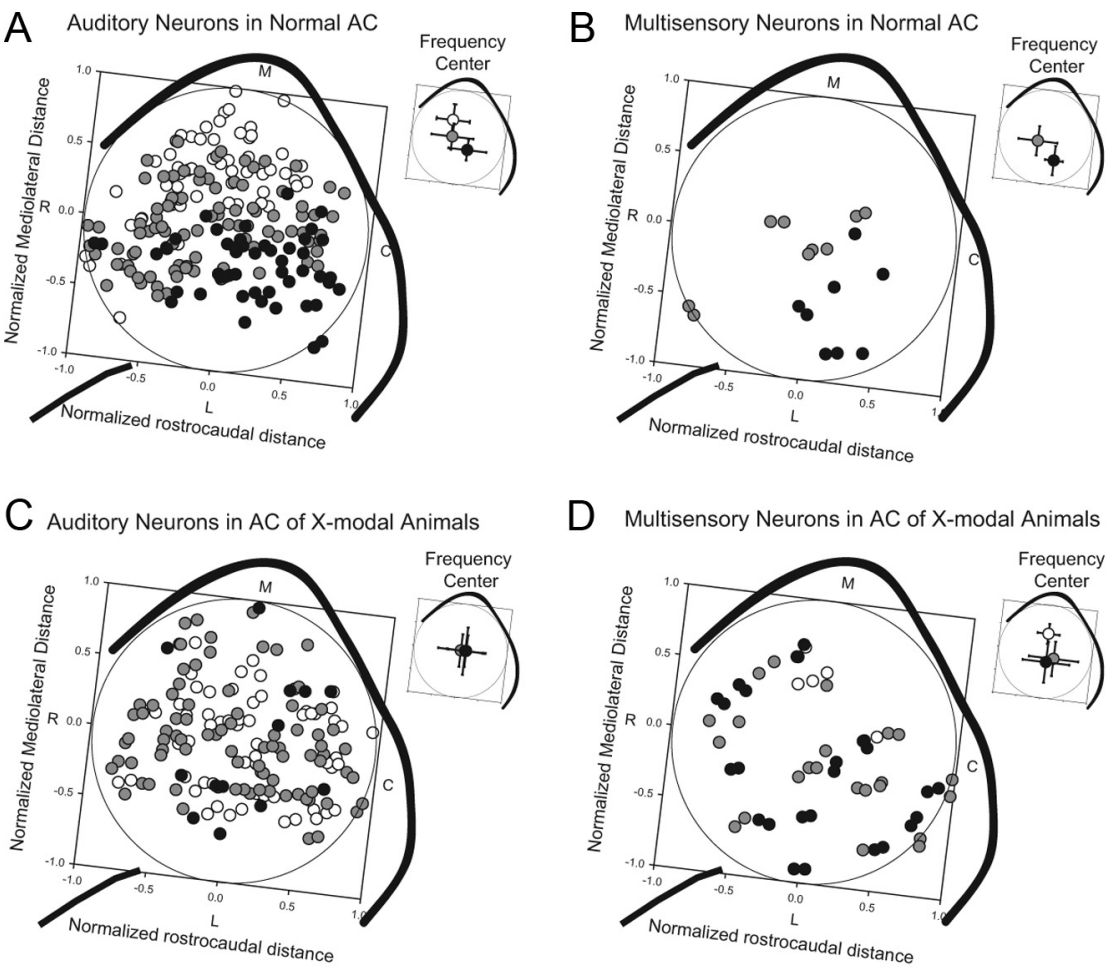

D Multisensory Neurons in AC of X-modal Animals

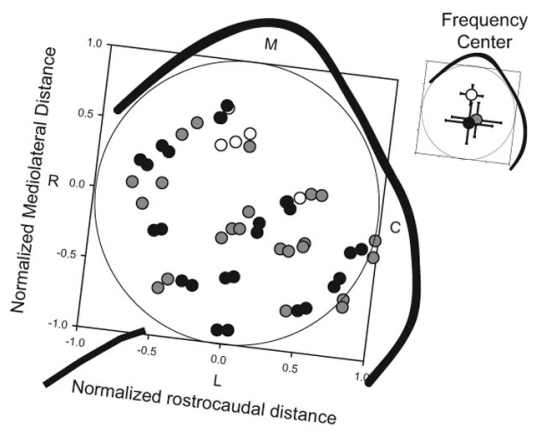

E Auditory Neurons in AC of Blind Animals

F

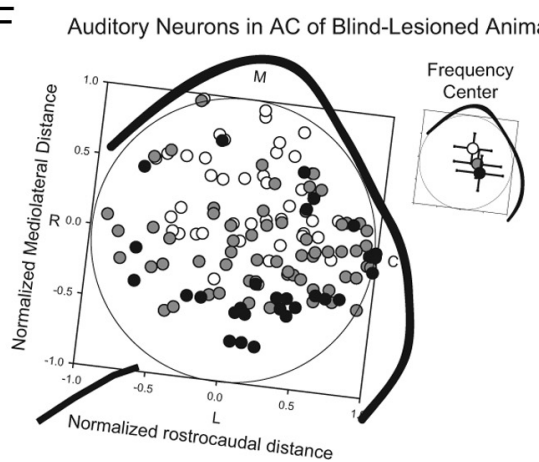

Figure 11. Distribution of frequency-tuned neurons in $\mathrm{AC}$ of ferrets using pooled data from each group. As in the micrographs, rostral is to the left and caudal to the right in these lateral views of the left AC. Open circles represent the class of high-frequency neurons. Gray circles represent mid-frequency neurons. Dark circles represent low-frequency neurons. The insets show the geographic centers $\pm S E$ of the distribution of each frequency class. $A$, Distribution of auditory neurons pooled from eight normal Distribution of multisensory neurons pooled from eight normal animals. These neurons are tuned appropriately for their location within the tonotopic map of auditory-only neurons. C, Distribution of auditory neurons pooled from nine XM animals. Note the lack otopic order and the overlap in distribution centers in the inset. D, Distribution of multisensory neurons pooled from nine XM animals. $\boldsymbol{E}$, Distribution of auditory neurons pooled from three blind animals. Tonotopy is normal. $\boldsymbol{F}$, Distribution of auditory neurons pooled from six blind-lesioned animals. Tonotopy is clear and the orientation of the map is normal.

Overall, our results show that basic auditory cortical response properties in the IC-lesioned, blind animals are essentially intact, independent of any contribution from the visual pathway. These results suggest that compensatory plasticity can substantially rebuild auditory function after deafferentation.

\section{Auditory function is improved in blind ferrets}

Many behavioral studies in humans and animal models have demonstrated that perceptual ability in the spared sensory modality is improved after blindness or deafness (Rauschecker and Korte, 1993; King and Parsons, 1999), perhaps as a result of XM plasticity. AC in deaf humans is recruited during visual processing (Finney et al., 2001), and the cortical area activated by sound 


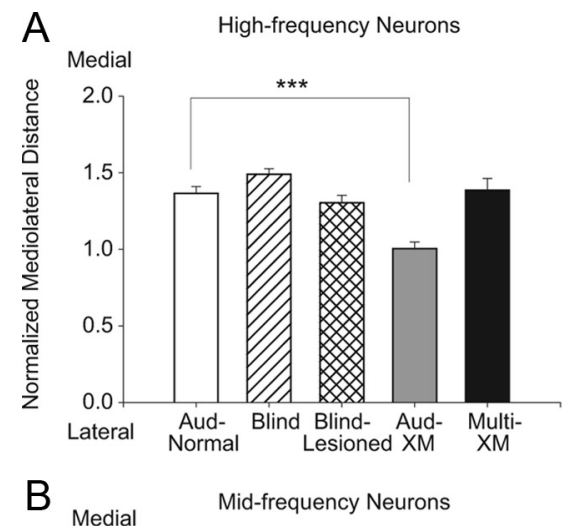

\section{Proportion}

A

High-frequency Neurons

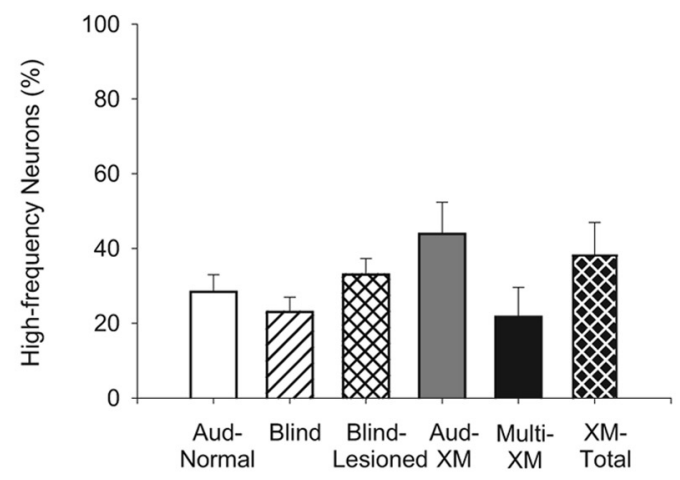

B Mid-frequency Neurons

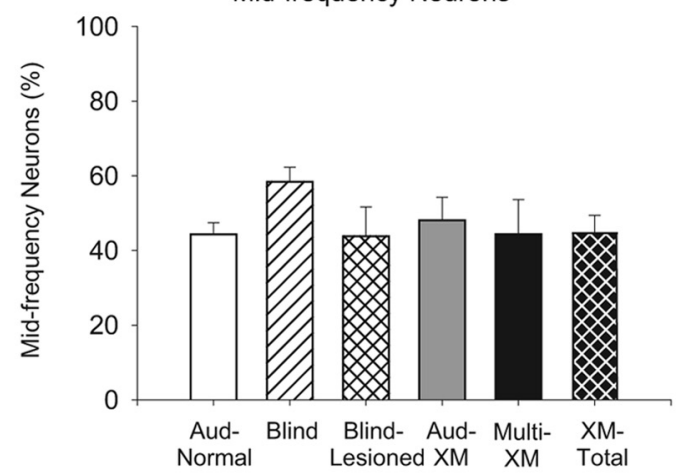

C Low-frequency Neurons

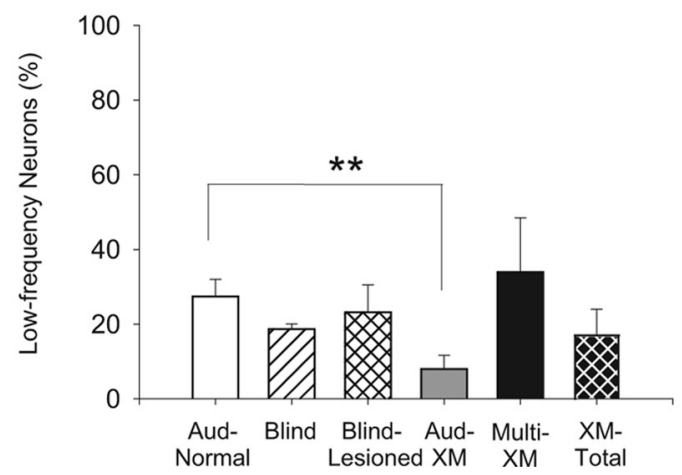

Figure 13. The proportion of frequency-tuned neurons in the five groups. $\boldsymbol{A}$, The $y$-axis represents the percentage of neurons tuned to high sound frequencies. There was no significant difference in the proportion of high-frequency neurons between groups. $\boldsymbol{B}$, There was no significant difference in the proportions of mid-frequency neurons between groups. $C$, The proportion of low-frequency neurons in the Aud-XM group was significantly lower than that in the normal group, but the proportion of low-frequency auditory and low-frequency multisensory neurons in the XM group considered together was not significantly different from that in the normal group. ${ }^{* *} p<0.01$.

The location of frequency-tuned neurons in the five groups. $A$, High-fr. neurons in XMAC were located significantly more laterally than those in normal AC. $\boldsymbol{B}$, The locations of mid-frequency neurons in the five groups were not significantly different from each other. C, Low-frequency neurons in XMAC were located significantly more medially than those in normal AC. $\boldsymbol{D}$, Low-, mid-, and high-frequency auditory neurons in the XM group were located at similar mediolateral locations (gray circle), showing a marked reduction in tonotopy compared with that in the normal group. Error bars show \pm SEM. ${ }^{*} p<0.05,{ }^{* * *} p<0.001$.

is expanded in blind humans (Elbert et al., 2002). Auditory response thresholds are also reduced in the AC of the early blind, suggesting that their auditory processing is more efficient (Stevens and Weaver, 2009). Auditory spatial tuning of neurons in the anterior ectosylvian cortical region of early-blind cats is sharpened (Korte and Rauschecker, 1993). Here we show that

auditory tuning characteristics in AC of blind animals are improved compared with normal, perhaps leading to improved perceptual ability as seen in blind and deaf patients. Thus, these ferrets may be a useful animal model for study of sensory substitution after deafness.

In conclusion, these findings provide additional evidence that, although AC can recover from neonatal deafferentation in some respects, its function is impaired by ectopic XM visual inputs, suggesting that minimization of XM activity may improve the 
chances of a successful outcome. Understanding the mechanism that underlies the compromise of auditory function will be important for facilitating recovery from brain damage, sensory/motor deafferentation, and sensory dysfunction and is the subject of a current study (Mao and Pallas, 2011).

\section{References}

Aitkin L, Irvine DRF, Webster WR (1984) Central neural mechanisms of hearing. In: Handbook of physiology: the nervous system III, pp 675-737. Bethesda, MD: American Physiological Society.

Bavelier D, Neville HJ (2002) Cross-modal plasticity: where and how? Nat Rev Neurosci 3:443-452.

Bizley JK, King AJ (2008) Visual-auditory spatial processing in auditory cortical neurons. Brain Res 1242:24-36.

Bizley JK, King AJ (2009) Visual influences on ferret auditory cortex. Hear Res 258:55-63.

Bizley JK, Nodal FR, Nelken I, King AJ (2005) Functional organization of ferret auditory cortex. Cereb Cortex 15:1637-1653.

Bizley JK, Nodal FR, Bajo VM, Nelken I, King AJ (2007) Physiological and anatomical evidence for multisensory interactions in auditory cortex. Cereb Cortex 17:2172-2189.

Brunso-Bechtold JK, Henkel CK (2005) Development of auditory afferents to the central nucleus of the inferior colliculus. In: The inferior colliculus (Winer JA, Schreiner CE, eds), pp 537-558. New York: Springer.

Buonomano DV, Merzenich MM (1998) Cortical plasticity: from synapses to maps. Annu Rev Neurosci 21:149-186.

Cacace AT (2003) Expanding the biological basis of tinnitus: crossmodal origins and the role of neuroplasticity. Hear Res 175:112-132.

Elbert T, Sterr A, Rockstroh B, Pantev C, Müller MM, Taub E (2002) Expansion of the tonotopic area in the auditory cortex of the blind. J Neurosci 22:9941-9944.

Finlay BL, Schneps SE, Schneider GE (1979) Orderly compression of the retinotectal projection following partial tectal ablation in the newborn hamster. Nature 280:153-155.

Finney EM, Fine I, Dobkins KR (2001) Visual stimuli activate auditory cortex in the deaf. Nat Neurosci 4:1171-1173.

Ghazanfar AA, Schroeder CE (2006) Is neocortex essentially multisensory? Trends Cogn Sci 10:278-285.

Harrison RV, Stanton SG, Ibrahim D, Nagasawa A, Mount RJ (1993) Neonatal cochlear hearing loss results in developmental abnormalities of the central auditory pathways. Acta Otolaryngol 113:296-302.

Harrison RV, Ibrahim D, Mount RJ (1998) Plasticity of tonotopic maps in auditory midbrain following partial cochlear damage in the developing chinchilla. Exp Brain Res 123:449-460.

Henkel CK, Keiger CJ, Franklin SR, Brunso-Bechtold JK (2007) Development of banded afferent compartments in the inferior colliculus before onset of hearing in ferrets. Neuroscience 146:225-235.

Herrmann K, Antonini A, Shatz CJ (1994) Ultrastructural evidence for synaptic interactions between thalamocortical axons and subplate neurons. Eur J Neurosci 6:1729-1742.

Insanally MN, Köver H, Kim H, Bao S (2009) Feature-dependent sensitive periods in the development of complex sound representation. J Neurosci 29:5456-5462.

Irvine DR (2000) Injury- and use-related plasticity in the adult auditory system. J Commun Disord 33:293-311.

Irvine DR, Rajan R, Brown M (2001) Injury- and use-related plasticity in adult auditory cortex. Audiol Neurootol 6:192-195.

Irvine DR, Rajan R, Smith S (2003) Effects of restricted cochlear lesions in adult cats on the frequency organization of the inferior colliculus. J Comp Neurol 467:354-374.

Izraeli R, Koay G, Lamish M, Heicklen-Klein AJ, Heffner HE, Heffner RS, Wollberg Z (2002) Cross-modal neuroplasticity in neonatally enucleated hamsters: structure, electrophysiology and behaviour. Eur J Neurosci 15:693-712.

Jastreboff PJ (1990) Phantom auditory perception (tinnitus): mechanisms of generation and perception. Neurosci Res 8:221-254.

Kaas JH (1991) Plasticity of sensory and motor maps in adult mammals. Annu Rev Neurosci 14:137-167.

Kakigi A, Hirakawa H, Harel N, Mount RJ, Harrison RV (2000) Tonotopic mapping in auditory cortex of the adult chinchilla with amikacin-induced cochlear lesions. Audiology 39:153-160.

Kamke MR, Brown M, Irvine DR (2003) Plasticity in the tonotopic organi- zation of the medial geniculate body in adult cats following restricted unilateral cochlear lesions. J Comp Neurol 459:355-367.

Kelly JB, Judge PW, Phillips DP (1986) Representation of the cochlea in primary auditory cortex of the ferret (Mustela putorius). Hear Res 24:111-115.

King AJ, Parsons CH (1999) Improved auditory spatial acuity in visually deprived ferrets. Eur J Neurosci 11:3945-3956.

Klinke R, Hartmann R, Heid S, Tillein J, Kral A (2001) Plastic changes in the auditory cortex of congenitally deaf cats following cochlear implantation. Audiol Neurootol 6:203-206.

Korte M, Rauschecker JP (1993) Auditory spatial tuning of cortical neurons is sharpened in cats with early blindness. J Neurophysiol 70:1717-1721.

Kral A (2007) Unimodal and cross-modal plasticity in the "deaf" auditory cortex. Int J Audiol 46:479-493.

Kral A, Sharma A (2012) Developmental neuroplasticity after cochlear implantation. Trends Neurosci 35:111-122.

Lee DS, Lee JS, Oh SH, Kim SK, Kim JW, Chung JK, Lee MC, Kim CS (2001) Cross-modal plasticity and cochlear implants. Nature 409:149-150.

Lomber SG, Meredith MA, Kral A (2010) Cross-modal plasticity in specific auditory cortices underlies visual compensations in the deaf. Nat Neurosci 13:1421-1427.

Lund RD, Lund JS (1976) Plasticity in the developing visual system: the effects of retinal lesions made in young rats. J Comp Neurol 169:133-154.

Mao YT, Pallas SL (2010) Invasion of ectopic visual inputs compromises auditory function in primary auditory cortex. Frontiers in Neuroscience Conference Abstract: 2010 South East Nerve Net and Georgia/South Carolina Neuroscience Consortium conferences, March 5-7, 2010, Atlanta.

Mao YT, Pallas SL (2011) Inhibition is increased in auditory cortex after invasion by ectopic visual inputs. Soc Neurosci Abstr 37:912.01.

Mao YT, Hua TM, Pallas SL (2011) Competition and convergence between auditory and cross-modal visual inputs to primary auditory cortical areas. J Neurophysiol 105:1558-1573.

Meredith MA, Lomber SG (2011) Somatosensory and visual crossmodal plasticity in the anterior auditory field of early-deaf cats. Hear Res 280:38-47.

Meredith MA, Stein BE (1983) Interactions among converging sensory inputs in the superior colliculus. Science 221:389-391.

Merzenich MM, Reid MD (1974) Representation of the cochlea within the inferior colliculus of the cat. Brain Res 77:397-415.

Merzenich MM, Kaas JH, Wall J, Nelson RJ, Sur M, Felleman D (1983) Topographic reorganization of somatosensory cortical areas $3 \mathrm{~b}$ and 1 in adult monkeys following restricted deafferentation. Neuroscience 8:33-55.

Middlebrooks JC, Bierer JA, Snyder RL (2005) Cochlear implants: the view from the brain. Curr Opin Neurobiol 15:488-493.

Moore DR, Hine JE (1992) Rapid development of the auditory brainstem response threshold in individual ferrets. Brain Res Dev Brain Res 66:229-235.

Moore DR, Semple MN, Addison PD (1983) Some acoustic properties of neurones in the ferret inferior colliculus. Brain Res 269:69-82.

Pallas SL, Finlay BL (1989) Conservation of receptive-field properties of superior colliculus cells after developmental rearrangements of retinal input. Vis Neurosci 2:121-135.

Pallas SL, Sur M (1994) Morphology of retinal axon arbors induced to arborize in a novel target, the medial geniculate nucleus. II. Comparison with axons from the inferior colliculus. J Comp Neurol 349:363-376.

Pallas SL, Littman T, Moore DR (1999) Cross-modal reorganization of callosal connectivity without altering thalamocortical projections. Proc Natl Acad Sci U S A 96:8751-8756.

Phillips DP, Judge PW, Kelly JB (1988) Primary auditory cortex in the ferret (Mustela putorius): neural response properties and topographic organization. Brain Res 443:281-294.

Piché M, Robert S, Miceli D, Bronchti G (2004) Environmental enrichment enhances auditory takeover of the occipital cortex in anophthalmic mice. Eur J Neurosci 20:3463-3472.

Popescu MV, Polley DB (2010) Monaural deprivation disrupts development of binaural selectivity in auditory midbrain and cortex. Neuron 65:718-731.

Rajan R (1998) Receptor organ damage causes loss of cortical surround inhibition without topographic map plasticity. Nat Neurosci 1:138-143.

Rajan R (2001) Plasticity of excitation and inhibition in the receptive field of 
primary auditory cortical neurons after limited receptor organ damage. Cereb Cortex 11:171-182.

Rajan R, Irvine DR, Wise LZ, Heil P (1993) Effect of unilateral partial cochlear lesions in adult cats on the representation of lesioned and unlesioned cochleas in primary auditory cortex. J Comp Neurol 338:17-49.

Rauschecker JP (1995) Compensatory plasticity and sensory substitution in the cerebral cortex. Trends Neurosci 18:36-43.

Rauschecker JP, Korte M (1993) Auditory compensation for early blindness in cat cerebral cortex. J Neurosci 13:4538-4548.

Röder B, Teder-Sälejärvi W, Sterr A, Rösler F, Hillyard SA, Neville HJ (1999) Improved auditory spatial tuning in blind humans. Nature 400:162-166.

Roe AW, Garraghty PE, Esguerra M, Sur M (1993) Experimentally induced visual projections to the auditory thalamus in ferrets: evidence for a W cell pathway. J Comp Neurol 334:263-280.

Schroeder CE, Smiley J, Fu KG, McGinnis T, O’Connell MN, Hackett TA (2003) Anatomical mechanisms and functional implications of multisensory convergence in early cortical processing. Int J Psychophysiol 50:5-17.

Servière J, Webster WR, Calford MB (1984) Isofrequency labelling revealed by a combined $\left[{ }^{14} \mathrm{C}\right]$-2-deoxyglucose, electrophysiological, and horseradish peroxidase study of the inferior colliculus of the cat. J Comp Neurol 228:463-477.

Sharma A, Gilley PM, Dorman MF, Baldwin R (2007) Deprivation-induced cortical reorganization in children with cochlear implants. Int J Audiol 46:494-499.

Sharma A, Nash AA, Dorman M (2009) Cortical development, plasticity and re-organization in children with cochlear implants. J Commun Disord 42:272-279.

Stein BE, Meredith MA (1993) The merging of the senses. Cambridge, MA: Massachusetts Institute of Technology.

Stevens AA, Weaver KE (2009) Functional characteristics of auditory cortex in the blind. Behav Brain Res 196:134-138.

Sur M, Garraghty PE, Roe AW (1988) Experimentally induced visual projections into auditory thalamus and cortex. Science 242:1437-1441.

Sutter ML, Schreiner CE (1991) Physiology and topography of neurons with multipeaked tuning curves in cat primary auditory cortex. J Neurophysiol 65:1207-1226.

Wallace MT, Meredith MA, Stein BE (1992) Integration of multiple sensory modalities in cat cortex. Exp Brain Res 91:484-488.

Wallace MT, Ramachandran R, Stein BE (2004) A revised view of sensory cortical parcellation. Proc Natl Acad Sci U S A 101:2167-2172.

Zhang LI, Bao S, Merzenich MM (2001) Persistent and specific influences of early acoustic environments on primary auditory cortex. Nat Neurosci 4:1123-1130.

Zhang LI, Bao S, Merzenich MM (2002) Disruption of primary auditory cortex by synchronous auditory inputs during a critical period. Proc Natl Acad Sci U S A 99:2309-2314. 